\title{
Flow Characteristics of the Drag Reducing Solid Wall ${ }^{*}$
}

\author{
Keizo WATANABE $^{* *}$ Satoshi OGATA ${ }^{* * *}$ Atushi HIROSE ${ }^{* * * *}$ \\ and Akihiro KIMURA ${ }^{* * * * *}$ \\ ** Department of Technology and risk Management, Tokyo University of Agriculture and Technology, \\ 2-24-16 Nakacho, Koganei-shi, Tokyo 184-8588, JAPAN \\ E-mail: mot1z019@cc.tuat.ac.jp \\ *** Department of Mechanical Engineering, Tokyo Metropolitan University, \\ 1-1 Minami Ohsawa, Hachiooji-shi, Tokyo 192-0397, JAPAN \\ E-mail: ogata-satoshi@c.metro-u.ac.jp \\ **** Yamaha Motor Co., Ltd., \\ 2500 Shingai, Iwata, Shizuoka 438-8501, JAPAN \\ E-mail: hirosea@yamaha-motor.co.jp \\ *****Department of Mechanical Engineering, Tokyo Metropolitan University, \\ 1-1 Minami Ohsawa, Hachiooji-shi, Tokyo 192-0397, JAPAN
}

\begin{abstract}
In order to clarify the laminar drag reduction and the slip velocity on a hydrophobic surface, the pressure loss of a rectangular duct was measured while applying various types of wall surface qualities to the duct inner wall. Test wall surfaces were classified into three types according to surface characteristics, which were a hydrophobic coating surface, a fine rectangular groove surface, and a fine rectangular groove with a hydrophobic coating. The directions of the fine rectangular groove are parallel, vertical, and random in the flow direction, and the widths are 5 and $10 \mu \mathrm{m}$. For fine rectangular grooves with a hydrophobic coating surface, it was clarified that the laminar drag reduction occurred in the Reynolds number range of $150<\mathrm{Re}<770$. The maximum drag reduction ratio is approximately $13 \%$ at $\mathrm{Re}=649$. The Reynolds number range in which the drag reduction occurred increased with decreasing width of the groove, and the direction of the grooves did not affect the drag reduction effect.
\end{abstract}

Key words: Internal Flow, Duct, Drag Reduction, Hydrophobic Wall, Pressure Loss, Flow Visualization

\section{Introduction}

Drag reduction phenomena in fluid flow have recently been examined from the viewpoint of energy savings in practical applications for engineering technology, and many studies have been conducted in this area of research. The goal of reducing the drag in a turbulent flow region is turbulence modification by flow control. This goal has been realized by adding drag reducing high molecular polymer or surfactant into liquid. Although the drag reduction method is restricted to the target of liquid flow, the riblet surface can be applied to liquid or gas flows in order to obtain the drag reduction. However, it is impossible to obtain a drag reduction in a laminar flow region by applying these techniques because turbulence does not exist in this flow region. Generally, flows related to industrial

\footnotetext{
${ }^{*}$ Received 19 Dec., 2006 (No. T-05-0272) Japanese Original : Trans. Jpn. Soc. Mech. Eng., Vol.71, No.712, B (2005), pp.2843-2848 (Received 16 Mar., 2005) [DOI: 10.1299/jee.2.108]
} 
applications are nearly turbulent. However, recently, the reduction of drag is important for the laminar flow region that occurs in a high viscosity liquid transport system or in very-low-order flow systems, such as those associated with micro-machines.

At the continuum hydrodynamics level, it is almost universally accepted that the boundary condition for a real fluid at a solid wall is the "no-slip" condition, i.e., the fluid velocity matches the velocity of the solid boundary. Thus, if fluid slip occurs at the solid wall, drag reduction can occur in the flow range. For laminar skin friction reduction, we reported the experimental results obtained for the friction factor of a circular pipe with a highly water repellent wall. ${ }^{(1)}$ The wall surface has a fractal-type microstructure with several fine grooves. It has been clarified that the apparent fluid slip velocity occurs at the highly water-repellent wall with the interface between gas and liquid. On the other hand, the existence of fluid slip has also been observed based on the pressure drop measurement ${ }^{(2)}$ of a hydrophobic glass tube and the velocity profile measurement ${ }^{(3)}$ of a micro-scale hydrophobic tube by a $\mu$-PIV system. Tyrell and Attard ${ }^{(4)}$ clarified the existence of micro bubbles on the hydrophobic wall in water by means of an atomic force microscope and reported that fluid slip occurred by means of these bubbles.

However, experiments have demonstrated the existence of two types of hydrophobic walls: drag reducing and non-drag reducing, for the same flow system size, despite of the high water repellency of these walls ${ }^{(5)}$. The discrepancy between these data is interpreted as indicative of new effects of flow on a hydrophobic wall. Thus, it is necessary to clarify the effect of the microstructure of the drag reducing hydrophobic wall on the fluid slip that occurs in the conventional flow system.

The purpose of the present study is to clarify experimentally the effect of the microstructure of the hydrophobic wall on the skin friction of a laminar flow in a flow system on the $10-\mu \mathrm{m}$-order scale by pressure measurement and flow visualization. The results reveal the friction factor and flow behavior near the wall of a channel flow.

\section{Experimental apparatus and method}

\section{1 Test Hydrophobic Wall}

Figure 1 shows micrographs ${ }^{(5)}$ of two types of highly water-repellent walls that have contact angles of approximately 150 degrees. The walls shown in Figs. 1(a) and (b) are the drag reducing and non drag reducing walls, respectively. The wall shown in Fig. 1(a) has several fine arranged-randomly grooves having widths of approximately $10 \mu \mathrm{m}$, and the wall shown in Fig. 1(b) is very rough but has no grooves. The grooves of the drag reducing wall were examined, and hydrophobic walls with the micro-structures shown in Fig. 2 were constructed. Figures 2(a) and (b) show parallel grooves in the axial flow direction and random $y$ print grooves, respectively. In Fig. 2(a), $p, w$ and $d$ are the pitch, width and the depth, respectively, of the grooves. Here, vertical grooves refer to grooves arranged vertically with respect to the axial flow direction. The grooves were made by the etching

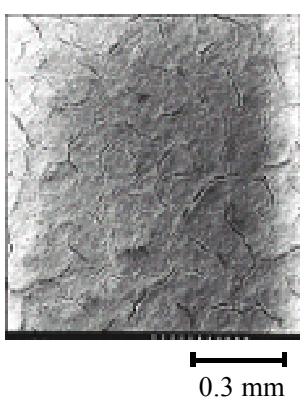

(a)

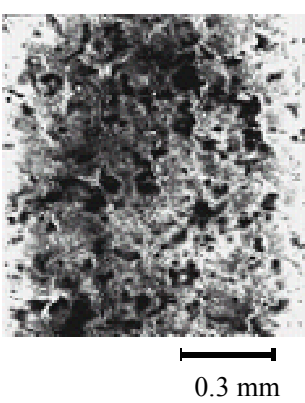

(b)

Fig. 1 Micrographs of the highly water-repellent wall 
Table 1 Dimensions of the test wall

\begin{tabular}{|c|c|c|c|c|c|c|c|}
\hline \multirow{2}{*}{ No } & \multirow{2}{*}{ Type } & \multirow{2}{*}{ Chemical treatment } & \multicolumn{5}{|c|}{ Groove } \\
\hline & & & Direction to flow & Width $(\mu \mathrm{m})$ & $\operatorname{Pitch}(\mu \mathrm{m})$ & $\operatorname{Depth}(\mu \mathrm{m})$ & Groove area $(\%)$ \\
\hline 1 & $\mathrm{~A}$ & HIREC X1 & & & - & & \\
\hline 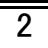 & & & parallel & $\overline{10}$ & 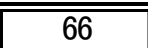 & 20 & $\overline{15}$ \\
\hline 3 & & & vertical & 10 & 66 & 20 & 15 \\
\hline 4 & & - & random & 10 & 66 & 20 & 15 \\
\hline 5 & B & - & parallel & 5 & 33 & 20 & 15 \\
\hline 6 & & & vertical & 5 & 33 & 20 & 15 \\
\hline 7 & & & random & 5 & 33 & 20 & 15 \\
\hline 8 & & & parallel & $\overline{10}$ & 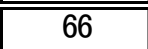 & 20 & 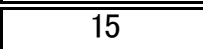 \\
\hline 9 & & & vertical & 10 & 66 & 20 & 15 \\
\hline 10 & 0 & ㅍㅁㄷ Х1 & random & 10 & 66 & 20 & 15 \\
\hline 11 & U & HUIEL XI & parallel & 5 & 33 & 20 & 15 \\
\hline 12 & & & vertical & 5 & 33 & 20 & 15 \\
\hline 13 & & & random & 5 & 33 & 20 & 15 \\
\hline
\end{tabular}

process on a silicon basis. The vertical grooves have widths of 5 and $10 \mu \mathrm{m}$, and the groove area ratio is determined as $15 \%$ for the total surface area by referring to the experimental results ${ }^{(5)}$. The dimensions of the test wall are listed in Table 1, and Types A, B, and C denote, respectively, a wall coated by a highly water-repellent spray only, a wall with several fine grooves, and a wall with several fine grooves coated by a highly water-repellent spray. A silica particle having a diameter of approximately $200 \mathrm{~nm}$ and a silicon resin are the main components in the treatment spray (HIREC X1, NTT-AT), and the surface condition is similar to the wall, as shown in Fig. 1(b). The drying time is approximately eight hours at $80^{\circ} \mathrm{C}$ using a dry oven. A micrograph of a wall coated by the treatment spray is shown in Fig. 3. The roughness of the wall is that of a hydraulic smooth wall. This was confirmed using a microscope for the Type $\mathrm{C}$ wall, for which the shape of the grooves is maintained after the coating.

Figures 4(a) and (b) show micrographs of $10-\mu \ell$ water droplets on a $5-\mu \mathrm{m}$ vertically grooved wall that was not coated with a highly water repellent spray and on a $5-\mu \mathrm{m}$ vertically grooved coated with a highly water repellent spray, respectively. As shown in Fig. 4(b), the water-repellency of the coated wall was high compared with that of the non-coated wall because the shape of the droplet is spherical.

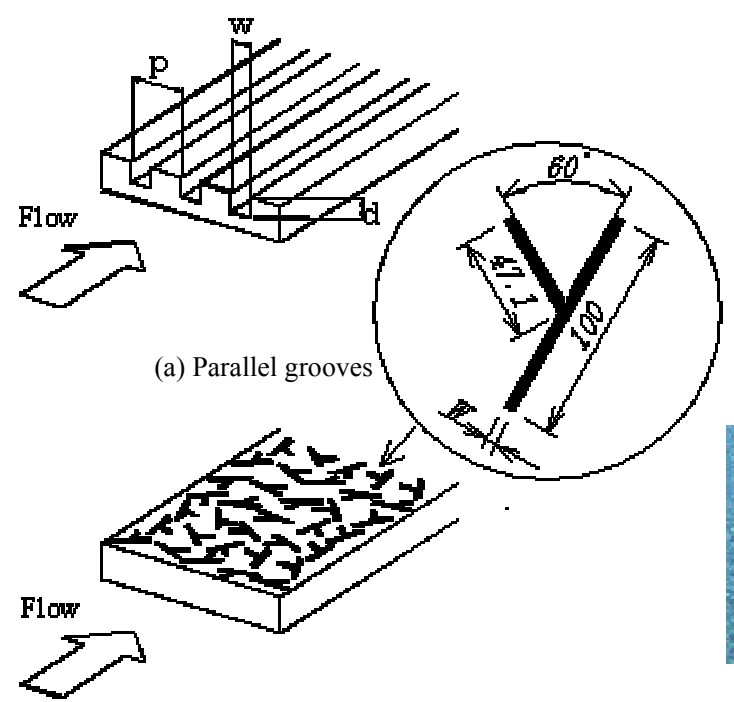

(b) Random grooves

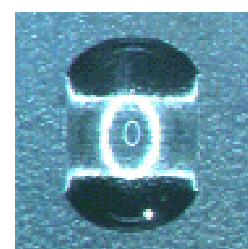

(a)No coating

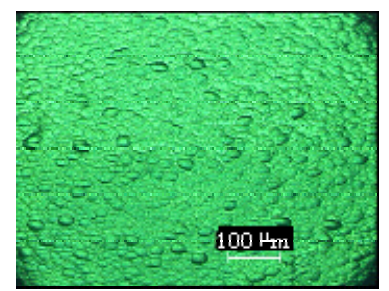

Fig. 3 Micrograph of test wall surface

Fig. 4 Micrographs of a $10-\mu l$ water drop on the test wall

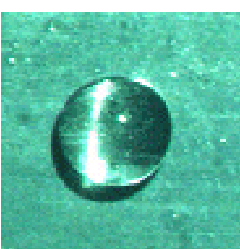

b)Coating with HIREC X1

Fig. 2 Idealized images of the test wall 


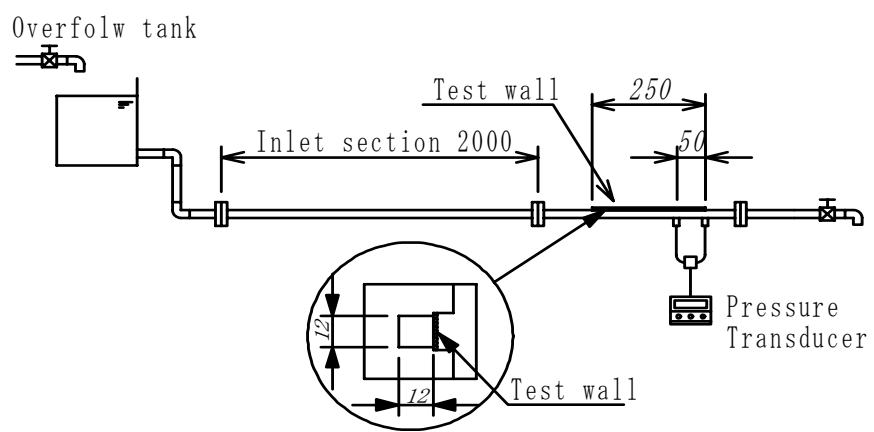

Fig. 5 Schematic diagram of the test pipe line system

\section{2 Skin Friction Measurement}

Measurement of the skin friction was performed by the measuring the pressure drop of a square duct, one side of which is made up of the test wall. Figure 5 shows a schematic diagram of a pipeline system, which consists of an inlet length and the test section. The test fluid is tap water, which flows in the test section at constant pressure using an over flow head tank. The flow system size is $12 \times 12 \mathrm{~mm}$ in the channel section. The pressure difference of $50 \mathrm{~mm}$ of duct length in the test section is measured by means of a pressure transducer (DP 103-06, Validain). The flow rate is varied by a control valve placed in the pipeline exit and is measured by means a dial scale. The reported pressure measurement at $\operatorname{Re}=150$ for the normal wall duct is the best estimate of the result, and with $95 \%$ confidence, the true value is believed to lie within $3.22 \%$ of the estimated result.

\section{Results and discussion}

The experimental results for the friction factor are shown in Fig. 6. In Fig. 6, $\lambda$ and Re are the friction factor and Reynolds number, respectively, of a channel flow. The experimental data for Type A and B walls agree well with the analytical results for a square duct obtained using the no fluid slip boundary condition and the Brasius formula for laminar and turbulent flow regions, respectively. However, the data for the Type $\mathrm{C}$ wall decrease slightly in the low Reynolds number range of Laminar flow, compared with the analytical results for the no-slip condition. According to the increase in the Reynolds number, the data agree with these formulas. In other words, drag reduction does not occur in this region. This tendency of the experimental data is recognized for both the vertical grooves and the grooves arranged randomly with respect to the flow direction.

We rearranged the experimental data of the Type $\mathrm{C}$ wall in the low Reynolds number range of $100<\operatorname{Re}<1,000$ in order to clarify the drag reduction phenomena by taking the product of $\lambda$ and Re. Figures 7(a) and (b) show the results for $10-\mu \mathrm{m}$ - and $5-\mu \mathrm{m}$-wide grooves, respectively, of the Type $\mathrm{C}$ wall. There are a few differences in the data obtained for the groove of different shapes. However, in the range of $\operatorname{Re}<700$, the 5 - $\mu \mathrm{m}$-wide groove experiences a drag reduction, compared to the $10-\mu \mathrm{m}$-wide groove. The maximum drag reduction ratio is approximately $13 \%$, as shown in Table 2 .

It is convenient to estimate the order of the friction factor if the analytical result is given. Watanabe et al. ${ }^{(1)}$ obtained the analytical result for the friction factor of a circular pipe with fluid slip by applying Navier's hypothesis ${ }^{(6)}$ as follows:

$$
\tau_{w}=\beta u_{s}
$$

where $u_{s}$ is the slip velocity at the wall, $\tau_{w}$ is the wall shear stress, and $\beta$ is the sliding coefficient. 


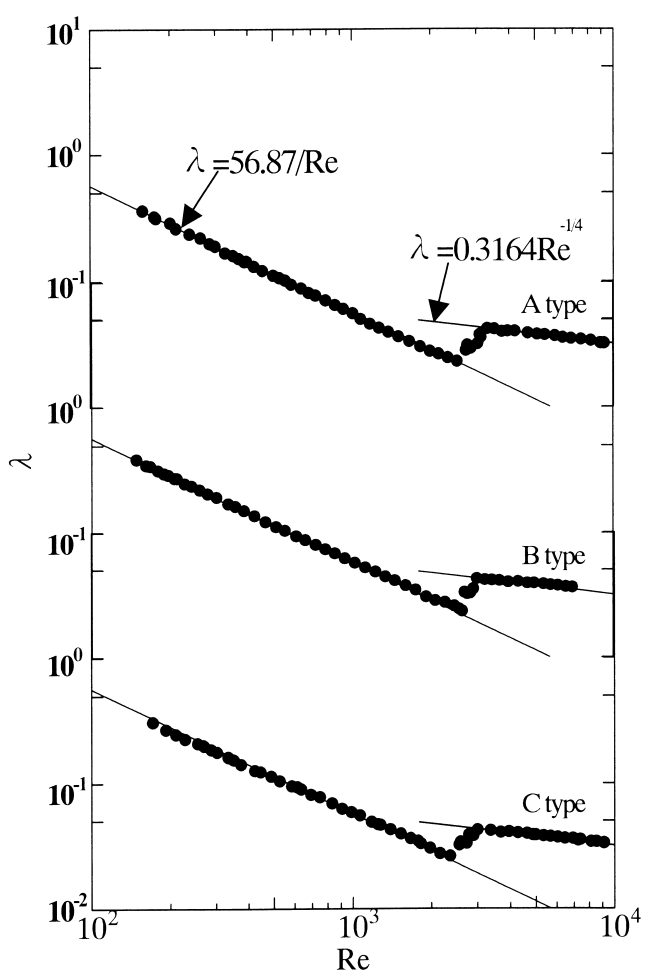

Fig. 6 Friction factor of the test wall (width of parallel grooves: $10 \mu \mathrm{m}$ )

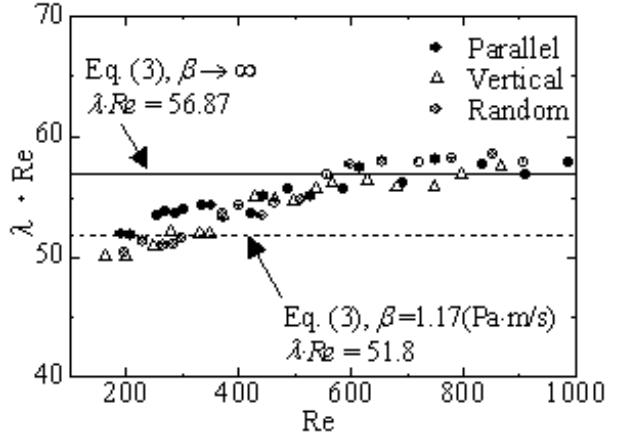

(a) Groove width: $10 \mathrm{~mm}$

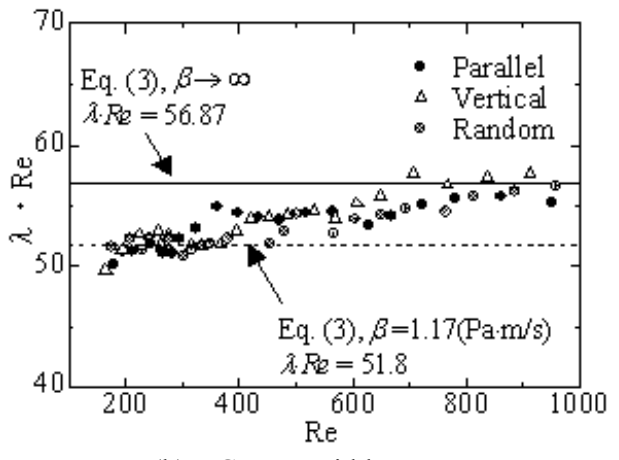

(b) Groove width: $5 \mathrm{~mm}$

Fig. 7 Friction factor of the test wall

Table 2 Drag reduction

\begin{tabular}{c|c|c|c}
\multicolumn{2}{c|}{ Groove } & \multicolumn{2}{c}{ Drag reduction } \\
\hline Direction & Width $(\mu \mathrm{m})$ & Maximum ratio(\%) & Re number range \\
\hline parallel & 10 & 8.8 & 526 \\
vertical & 10 & 11.9 & 496 \\
random & 10 & 11.4 & 512 \\
parallel & 5 & 11.8 & 723 \\
vertical & 5 & 12.6 & 649 \\
random & 5 & 10.6 & 764
\end{tabular}

By assuming the incompressible steady and fully developed flow in a duct, the Navier-Stokes equation in Cartesian coordinates is as follows:

$$
\frac{\partial w^{2}}{\partial^{2} x}+\frac{\partial w^{2}}{\partial^{2} y}=\frac{1}{\mu} \frac{d p}{d z}
$$

where $w$ is the axial velocity, $p$ is the pressure, and $\mu$ is the viscosity of fluid. We can reduce the axial velocity $w$ by applying the boundary condition of Eq. (1) to Eq. (2).

Finally, the analytical result for the friction factor $\lambda$ of a duct with fluid slip at one side wall, as shown in Fig. 5, is obtained as: 


$$
\lambda=\frac{64}{\operatorname{Re}} \frac{\pi^{4} \varepsilon^{3}}{64(1+\varepsilon)^{2}} \frac{1}{\sum_{n} \frac{1}{n^{4}}\left[\frac{1}{n \pi}\left\{2 \frac{\cosh n \pi \varepsilon-1}{\sinh n \pi \varepsilon}-\frac{\frac{(\cosh n \pi \varepsilon-1)^{2}}{\sinh n \pi \varepsilon}}{\frac{2 a}{n \pi} \frac{\beta}{\mu} \sinh n \pi \varepsilon+\cosh n \pi \varepsilon}\right\}-\varepsilon\right]}
$$

$$
\mathrm{n}=1,3,5,7, \cdots \cdots
$$

where $\varepsilon$ is the aspect ratio of the duct. The aspect ratio of a duct for which the width and height of the section are defined as $x=2 a$ and $y=2 b$ is given as $\varepsilon=(a / b)$. For a square duct, $\varepsilon=1$.

In general, although it is very difficult to estimate the value of the sliding constant, $\beta$, because it depends on the physical properties of the wall and fluid, we can analogize the value based on previous research ${ }^{(1)}$. If we substitute $\beta=1.17$ (Pa.s/m) into Eq. (2), the analytical result is obtained as $\mathrm{Re} \cdot \lambda=51.8$, as shown by the dotted line in Fig. 7. The analytical result agrees well with the experimental data in the low Reynolds number range. However, this does not fit the data in the large Reynolds number region because the experimental data increases with increasing Reynolds number.

As mentioned above, the gas interface in the groove is important for the occurrence of an apparent fluid slip at the wall. The decrease of the drag reduction ratio with the increase in the Reynolds number is considered to be caused by the varnish on the interface in the grooves. It is therefore necessary to experimentally clarify the behavior of the gas interface in the groove in order to clarify the drag reduction phenomena.

Figure 8 shows the experimental apparatus used for flow visualization. In Fig. 8, a 5 - $\mu \mathrm{m}$-wide test wall, which is vertical with respect to the flow direction, is arranged as the upper wall of the flow channel. The flow channel is a $12 \times 6 \mathrm{~mm}$ duct in the flow system. The test wall is constructed of glass coated by a highly water repellent spray after the etching process. The case when no coating was applied to the wall was also investigated to determine the difference that the coating made. The light source is positioned on upper the wall, and photographs are taken by means of a microscope.

The visualization photographs are shown in Figs. 9(a) (c). The walls shown in Figs. 9(a) and (b) correspond to Type $\mathrm{B}$ and Type $\mathrm{C}$ in Table 1, which are non-drag reducing and drag reducing walls, respectively, in the listed Reynolds number range. At $\mathrm{Re}=150$, white stripes are recognized in Fig. 9(b), but they are not seen in Fig. 9(a). The pitch of the stripes agrees with that of the grooves. As the Reynolds number increases, the white stripes, seen in Fig. 9(b), become broken, as shown in Fig. 9(c). The stripes clearly indicate the gas interface in the grooves, and they are broken by the increase in the wall shear stress.

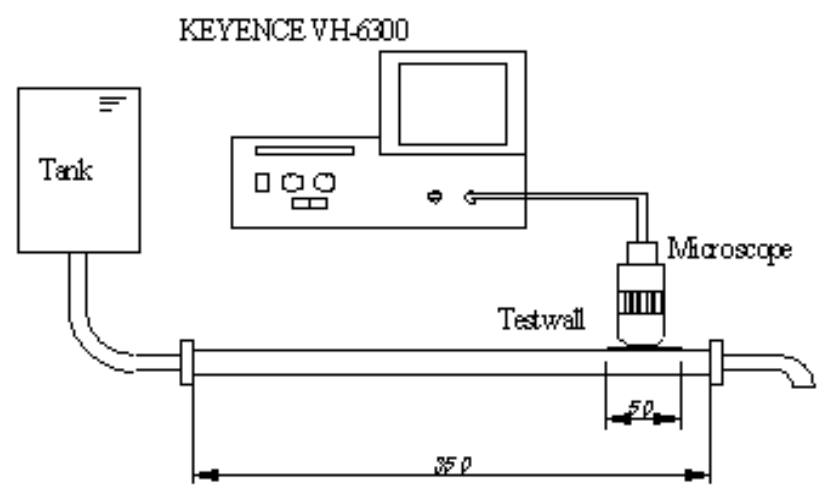

Fig. 8 Schematics of visualization devices 


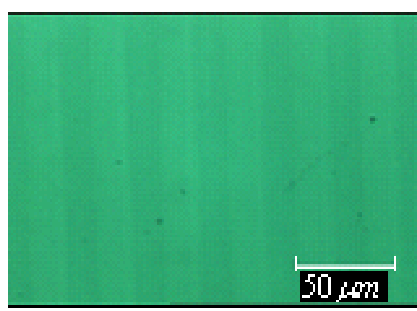

(a) No coating $\left(\mathrm{Re}=150, \tau_{\mathrm{w}}=0.042 \mathrm{~Pa}\right)$

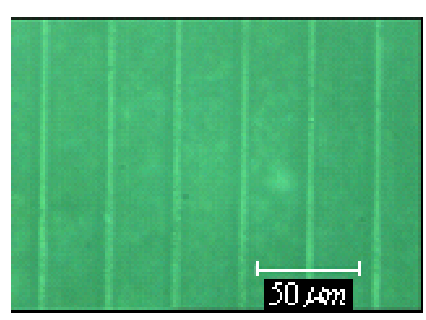

(b) Coating

$\left(\mathrm{Re}=150, \tau_{\mathrm{w}}=0.042 \mathrm{~Pa}\right)$

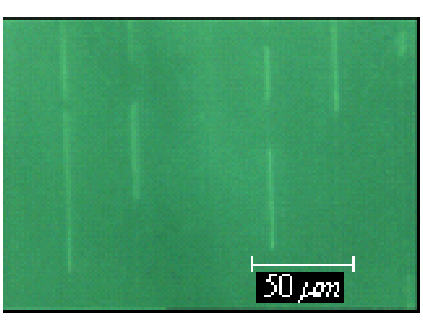

(c) Coating $\left(\mathrm{Re}=570, \tau_{\mathrm{w}}=0.168 \mathrm{~Pa}\right)$

Fig. 9 Visualization photograph $(5 \mu \mathrm{m}$ width vertical groove $)$

Consequently, the gas interface is important in generating the apparent fluid slip at the hydrophobic wall with a micro structure.

\section{Summary}

Three types of walls with micro structure were constructed by an etching process and the skin friction was measured using a channel flow system in order to clarify the drag reduction phenomena with the apparent fluid slip at the walls. Drag reduction was observed in the friction factor of the square duct with one side wall coated with a highly water-repellent spray in the range of $150<\operatorname{Re}<770$, and the maximum drag reduction ratio was approximately $13 \%$. The $5-\mu \mathrm{m}$-wide grooves were effective for drag reduction for a flow system of this scale. The visualization results indicated that the gas interface in the grooves is retained for the wall coated with a highly water-repellent spray, but not for the non-coated wall. Since the drag reduction does not occur in the region without the interface, the gas interface is a necessary component in this phenomena. The agreement between the experiments and the analytical solution obtained from a macroscopic viewpoint using the fluid slip boundary condition was good.

\section{Acknowledgements}

This research was supported by a Grant-in Aid for Research (B) (No. 15360099) from the Japan Society for the Promotion of Science (JSPS).

\section{References}

(1) Watanabe, K., Yanuar, and Udagawa, H., Drag Reduction of Newtonian Fluid in a Circular Pipe with a Highly Water-repellent Wall, Journal of Fluid Mechanics, Vol. 381(1999), pp. 225-238.

(2) Ruckenstein, E. and Rajora, P., On the No-Slip Boundary Condition of Hydrodynamics, Journal of Colloid and Interface Science, Vol. 96(1983), pp. 488-491.

(3) Tretheway, D.C. and Meinhart, C.D., Apparent Fluid Slip at Hydrophobic Microchannel Walls, Physics of Fluids, Vol. 14, No. 3(2002), pp. 9-12.

(4) Tyrell, J.W. and Attard, P., Images of Nanobubbles on Hydrophobic Surface and their Interactions, Physical Review Letters, Vol. 87, Issue 17(2001), 176104.

(5) Watanabe, K., Takayama, T., Ogata, S., and Isozaki, S., Flow between Two Coaxial Rotating Cylinders with Highly Water-Repellent Wall, AIChE Journal, Vol. 49, No. 8(2003), pp. 1956-1963.

(6) Navier. C.L.M.H., (Goldstein, S., Modern Developments in Fluid Dynamics (1965), 677-680, Dover Publication, Inc., New York.) 